\title{
Diagnostic d'une anéjaculation
}

\author{
Antoine LEMAIRE
}

CETPARP, Lille

RESUME

L'anéjaculation correspond à l'absence d'éjaculation durant l'orgasme.

La plupart des anéjaculateurs sont conscients de leur anomalie mais certains sujets peu avertis consultent pour infertilité et l'anéjaculation est suspectée lors d'un essai de spermogramme.

Il faut donc interroger l'homme sur sa capacité à éjaculer par exemple durant le sommeil, par masturbation ou, moins fréquemment, selon les positions coïtales.

Il existe différentes variétés d'anéjaculations.

L'interrogatoire est certainement le temps le plus important du diagnostic et ce diagnostic repose sur l'analyse de la séméiologie sexuelle et de l'orgasme. II est primordial de définir le caractère primaire ou secondaire du trouble.

Lorsque le diagnostic d'anéjaculation est confirmé et également différencié de l'éjaculation rétrograde et de l'éjaculation asthénique, il convient de différencier l'orgasme sans éjaculation où il n'y a pas non plus d'éjaculation normale en dehors du coït, de l'anéjaculation sans orgasme où existe une éjaculation normale en dehors du coït. Les causes peuvent se répartir en causes organiques et causes psychologiques.

Les principaux facteurs psychologiques susceptibles de jouer un rôle en cas d'anéjaculation sans orgasme peuvent se répartir en six catégories. Il faut toutefois insister sur le fait que la présence d'un de ces facteurs chez l'anéjaculateur n'implique pas qu'il soit obligatoirement la cause de la dysfonction sexuelle ; le diagnostic d'origine psychogène doit rester un diagnostic d'élimination après exclusion des causes organiques possibles.

Mots clés : éjaculation, anorgasmie, éjaculation rétrograde, hypofertilité

\section{INTRODUCTION}

L'anéjaculation correspond à l'absence d'éjaculation par le méat urétral malgré une stimulation sexuelle appropriée.

Trois variétés d'anéjaculation sont ainsi définies :

- Ejaculation rétrograde : elle est liée à un défaut de fermeture du sphincter interne de la vessie, d'où émission et éjaculation rétrograde dans la vessie.

- Orgasme sans éjaculation ou éjaculation sèche.

- Anéjaculation sans orgasme ; le terme éjaculation retardée est réservée aux cas où l'éjaculation est obtenue mais après un délai anormalement prolongée.

\section{Qui consulte pour anéjaculation?}

- Un couple lors d'un bilan de stérilité.

- L'homme qui constate qu'il n'y a jamais ou plus d'éjaculation lors des rapports. Le caractère primaire ou secondaire du trouble est un élément important à définir, le pronostic étant différent en fonction des cas.

- Un homme plus âgé qui constate une baisse des sensations orgasmiques avec une perte progressive de l'éjaculation (Déficit Androgénique Lié à l'Age, DALA) lors des rapports sexuels.

\section{L'interrogatoire}

L'interrogatoire est le temps essentiel de la consultation. II permet de mener une enquête étiologique en faisant préciser :

- Les antécédents de chirurgie abdominopelvienne, de pathologies neurologiques, de diabète.

\section{Correspondance :}

Dr Antoine LEMAIRE - 3 Allée Carolus, 59000 Lille - Email lemaire-antoine@wanadoo.fr 
- La prise d'une traitement médicamenteux éventuel.

- L'existence d'autres anomalies mictionnelles pouvant révéler une neuropathie urogénitale.

- Les paramètres de la fonction sexuelle :

- l'intérêt sexuel dont la baisse évoquerait une endocrinopathie ;

- la qualité des érections et leur maintien ;

- les circonstances d'apparition de l'anéjaculation et le caractère primaire ou secondaire ;

- en cas d'anéjaculation sans orgasme, le contexte éducatif, psychologique et conjugal.

Le diagnostic est retenu lorsque les critères suivants sont réunis [1]:

- absence d'éjaculation par le méat urétral au cours de la plupart des essais,

- en dépit d'une érection normale,

- et d'une stimulation sexuelle appropriée et suffisamment prolongée.

\section{Examen clinique}

L'examen clinique doit toujours comporter un examen des organes génitaux externes et un toucher rectal. II permet ainsi une évaluation de l' imprégnation androgénique et des caractères sexuels secondaires.

Une asthénie physique et psychique associée à une baisse de l'intérêt sexuel, des difficultés d'érection, une diminution de la masse et de la force musculaire ainsi que de la pilosité sont symptomatiques de l'hypogonadisme.

Un examen neurologique minimum pour vérifier la sensibilité du testicule et du périnée, les réflexes cutanés abdominaux et crémastériens, les réflexes ostéotendineux des membres inférieurs et le reflexe cutané plantaire.

\section{Diagnostic des anéjaculations}

Le Tableau 1 résume les caractéristiques des différents types d'anéjaculation.

Pour différencier l'orgasme sans éjaculation de l'éjaculation rétrograde, il est nécessaire de réaliser un examen des urines après un rapport sexuel à la recherche de spermatozoïdes, ou de phosphatases acides prostatiques en cas d'éjaculation rétrograde avec azoospermie.

Tableau 1 : Les différents types d'anéjaculation.

\begin{tabular}{|c|c|c|}
\hline & $\begin{array}{l}\text { Ejaculation hors } \\
\text { du coït }\end{array}$ & Orgasme \\
\hline Orgasme sans éjaculation & Non & Oui \\
\hline Anéjaculation sans orgasme & Oui (nocturne) & Non \\
\hline Ejaculation rétrograde & Non & Oui \\
\hline
\end{tabular}

\section{LES CAUSES DE L'ANÉJACULATION}

Elles peuvent se répartir comme suit [2] : anomalies congénitales, causes post traumatiques, problèmes infectieux, problèmes neurologiques, pathologies endocriniennes, origine iatrogène et cause psychologique.

\section{Anomalies congénitales}

L'agénésie épididymo-déférentielle peut laisser persister un éjaculat de volume très réduit. Dans certains cas, des canaux éjaculateurs peuvent être obstrués pas des kystes issus des vestiges mullériens et se vident parfois dans ces kystes.

\section{Causes post traumatiques}

Un délabrement musculaire avec atteinte du sphincter interne de la vessie peut être provoqué par résection du col vésical ou prostatectomie simple.

Un délabrement nerveux avec atteinte des fibres sympathiques peut être provoqué par une chirurgie vasculaire (endartériectomie, sympathectomie lombaire), un curage ganglionnaire (cancer du testicule), une chirurgie colorectale, ou une chirurgie de malformations pelviennes.

Un délabrement mixte (musculaire, nerveux, canaux éjaculateurs) est observé dans la chirurgie du cancer de la prostate et du cancer de la vessie.

\section{Problèmes infectieux}

Une infection génitale ou une urétrite peut produire une obstruction à tout niveau du tractus urogénital surtout lorsque le traitement a été mis en place tardivement. La tuberculose génitale peut également produire des lésions à ce niveau ainsi que la schistosomiase largement présente en Afrique [3] ; dans ce dernier cas, il est souvent associé une hémospermie.

\section{Problèmes neurologiques}

On peut les répartir en myélopathies et en neuropathies périphériques.

\section{a) les myélopathies}

- non traumatiques : sclérose latérale amyotrophique, sclérose en plaques, syringomyélie, spina bifida ;

- traumatiques : section médullaire plus ou moins complète.

\section{b) les neuropathies périphériques}

- secondaires à une complication d'un diabète ;

- post traumatiques : fracture du bassin.

\section{Pathologies endocriniennes}

L'anéjaculation en relation avec une endocrinopathie est souvent associée à d'autres symptômes sexuels tels que baisse de l'intérêt sexuel et troubles de l'érection.

Un hypoandrisme peut être responsable d'une éjaculation 
retardée, d'une anéjaculation sans orgasme ou d'un orgasme sans éjaculation par aspermie.

Quelques cas d'hyperprolactinémie ont été rapportés comme étant à l'origine d'une anéjaculation.

\section{Origine iatrogène}

Les IMAO augmentent le taux de norepinéphrine, épinéphrine, dopamine, sérotonine ; ils peuvent ainsi être responsables d'une éjaculation retardée ou absente.

Les benzodiazépines peuvent inhiber l'éjaculation probablement en augmentant le GABA (acide gamma aminobutirique).

Les antidépresseurs sérotoninergiques : des substances telles que la clomipramine, la fluoxétine, la paroxétine, la sertraline inhibent la recapture de la sérotonine et sont souvent responsables d'éjaculation retardée voire absente.

\section{Causes psychologiques}

- Facteurs éducatifs : une éducation trop stricte, une ignardise sexuelle.

- Technique masturbatoire inhabituelle.

- Sentiment de crainte (crainte ou refus de féconder la partenaire, crainte d'exposer son insuffisance de virilité ou de fertilité), de dégoût, de culpabilité du plaisir sexuel associé à l'acte d'éjaculer.

- Tendance générale de la personnalité à rationaliser, intellectualiser et à contrôler les émotions, d'où une difficulté à se laisser aller à ses sensations.

- Problèmes interpersonnels tels que conflit conjugal, ambivalence vis à vis de la partenaire.

- Conditionnement d'une réponse d'évitement.

\section{LES EXPLORATIONS COMPLÉMENTAIRES SONT-ELLES NECESSAIRES ?}

L'organigramme de la Figure 1 peut résumer la conduite à tenir.

Lors d'une revue récente, Delavierre [4] a rapporté une série de 72 cas d'anéjaculation :

Dans $1 / 3$ des cas (25 patients), aucun bilan n'était nécessaire.

Un bilan biologique fut effectué chez 45 patients : la glycémie était normale dans tous les cas et 6 hypogonadismes furent dépistés.

Des explorations neurophysiologiques pelvipérinéales furent effectuées chez 12 patients et 6 fois s'averaient pathologiques, mais dans chaque cas la neuropathie était connue ou suspectée.

Des explorations morphologiques furent réalisées 8 fois (échographie endorectale $n=5$, fibroscopie urétro-vésicale $n=3$ ) et n'ont mis en évidence d'anomalie qu'une seule fois.

Ces données concordent avec une étude précédente, où dans 60 cas d'anéjaculations sans orgasme aucune cause organique n'avait été trouvée parmi les 36 cas primaires. En revanche parmi les cas secondaires, 9 cas sur 24 résultaient d'une cause organique qui, 8 fois sur 9 , pouvait être détectée par un interrogatoire et un examen clinique complet.

\section{CONCLUSION}

L'anéjaculation reste un motif régulièrement évoqué lors des consultations de sexologie et de stérilité.

L'anéjaculation sans orgasme est le plus souvent d'origine psychogène.

Les différentes causes organiques peuvent induire

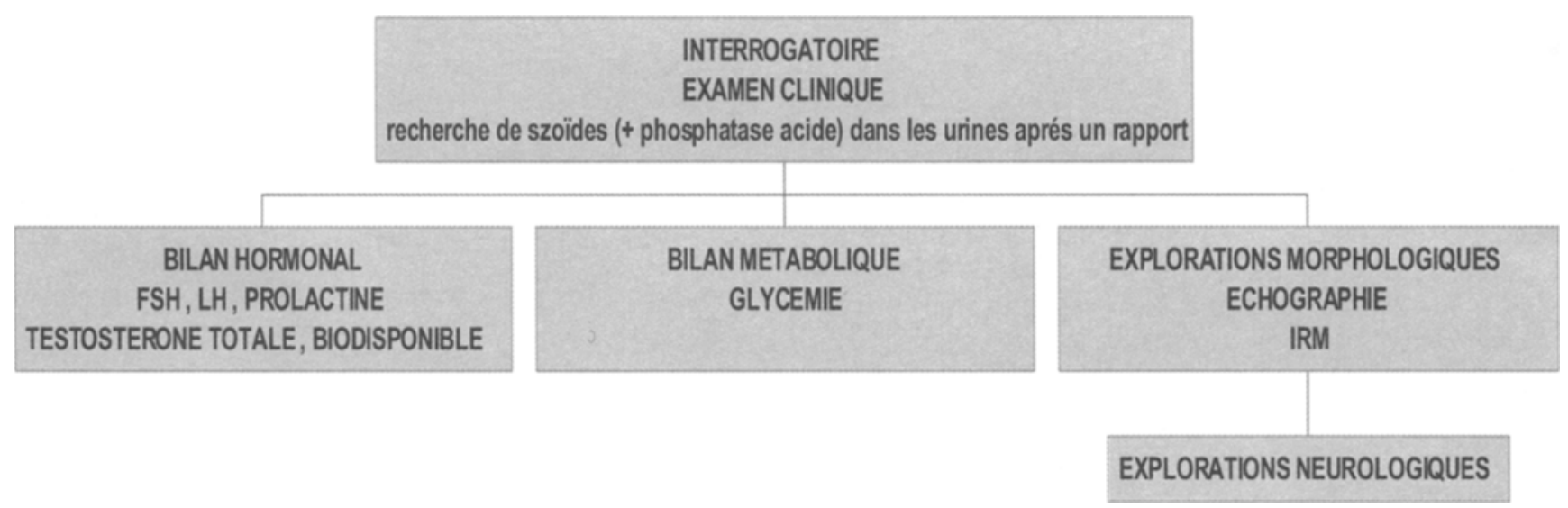

Figure 1: Conduite à tenir devant une anéjaculation 
indifféremment des anéjaculations avec ou sans orgasme.

Lors de la première consultation, l'interrogatoire est un temps crucial pour définir le contexte et le caractère primaire ou secondaire du trouble.

Avant d'envisager des moyens mécaniques visant à forcer le symptôme, tel que le vibromasseur, un essai de traitement psychothérapique et sexothérapique doit être mis en place.

\section{REFERENCES}

1. BUVAT J. : Perturbations balistiques de l'éjaculation. In : Buvat J., Jouannet P. eds. L'éjaculation et ses perturbations. Paris, Simep, $1984:$ 35-98.

2. JARDIN A. : L'éjaculation : aspects physiologiques et pathologiques. In : La voie séminale et ses glandes : leur rôle dans l'infertilité. Progrès en Andrologie 5. Paris, Doin, 1994 : 79-89.

3. HENDRY W.F. : Male orgasmic and ejaculatory disorders. In :

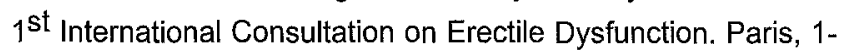
3 juillet 1999 ; Committee $13: 477-506$.

4. DELAVIERRE D. : Diagnostic d'une anéjaculation : à propos de 72 cas. $2^{\text {ème }}$ forum méditerranéen d'andrologie ; Collioure 12 14 juin 2003.

Communication au $\mathrm{XX}^{\circ}$ Congrès de la Société d'Andrologie de Langue Française, Orléans, 11-13 décembre 2003.

Manuscrit reçu : janvier 2004 ; accepté : février 2004

\section{ABSTRACT}

Diagnosis of anejaculation

Antoine LEMAIRE

Anejaculation corresponds to absence of ejaculation during orgasm. Most men with anejaculation are aware of their abnormality, but some poorly educated subjects consult for infertility and anejaculation is only suspected on the sperm count. The patient must therefore be questioned about his capacity to ejaculate, for example while sleeping, by masturbation or, less frequently, according to various coital positions. There are various types of anejaculation. Clinical interview is certainly the most important phase of the diagnostic work-up, which is based on analysis of sexual signs and orgasm. It is essential to define the primary or secondary nature of the disorder. After confirming the diagnosis of anejaculation and distinguishing it from retrograde ejaculation and asthenic ejaculation, orgasm without ejaculation and without normal ejaculation outside of coitus must be distinguished from anejaculation without orgasm but with normal ejaculation outside of coitus. The causes of anejaculation may be organic and psychological. The main psychological factors likely to play a role in anejaculation without orgasm can be classified into six categories. However, the presence of one of these factors in a patient with anejaculation does not necessarily imply that it is the cause of the sexual dysfunction; the diagnosis of psychogenic anejaculation is a diagnosis of exclusion after all possible organic causes have been eliminated.

Key words: ejaculation, anorgasmia, retrograde ejaculation, hypofertility 